Ann. Biol. anim. Bioch. Biophys., 1978, 18 (5), 1197-1203.

\title{
Étude, chez le rat, de la fonction pituitaire gonadotrope de femelles dysgénésiques jusqu'à 12 mois d'âge
}

par Elizabeth VANHEMS ${ }^{(1)}$, Jacqueline BOUSQUET, D. VALERO *, M. P. DUBOIS **

Laboratoire d'Histologie ef Embryologie, U.E.R. 3.

Université Bordeaux II, 146, rue Léo-Saignat, 33076 Bordeaux.

* INSERM - U 53, Domaine de Correire, 33077 Bordeaux.

** Station de Physiologie de la Reproduction, INRA, 37380 Monnaie.

Summary. Gonadotropic cells in dysgenesic females : Immunocytology and radioimmunoassay of $L H$ in 1 to 12-month old rats.

Using LH immunofluorescence and radioimmunoassay, pituitary gonadotropic activity has been studied in 1 to 12 -month old female rats with ovarian dysgenesy due to early destruction of germ cells by a radiomimetic, the Misulban. Immnunofluorescence using an anti-porcine $\beta$-LH antiserum on pituitaries of 48 to 210 -day old females demonstrated extensive gonadotropic cell hypertrophy ; plasma LH levels also increased sharply. However, in 1-year old females this cell type exhibited a marked regression and LH levels decreased. These results indicate gonadotropic hyperactivity in the dysgenesic females, as after castration, and a significant decrease of this activity when the animals age.

\section{Introduction.}

La dysgénésie ovarienne expérimentale, chez la Ratte, entraîne la présence de taux élevés de gonadotropine circulante, mis en évidence par la technique histophysiologique des greffes (Vanhems ef Bousquet, 1975b) ; nous avons complété cette étude par la réalisation de dosages radioimmunologiques de $\mathrm{LH}$ plasmatique et nous avons recherché si cette élévation s'accompagnait de changements au niveau de l'hypophyse.

\section{Matériel et méthodes.}

Les Rattes dysgénésiques, de souche Wistar, sont issues de mères traitées, au $15^{e}$ jour de leur gestation, par une injection intra-péritonéale de Misulbañ $(10 \mathrm{mg} / \mathrm{kg})$ (Vanhems et Bousquet, 1971, 1973). Les animaux sont élevés dans un local à tempérafure constante $\left(25^{\circ} \mathrm{C}\right)$, éclairé artificiellement et régulièrement $(14 \mathrm{~h}$ de lumière pour $10 \mathrm{~h}$ d'obscurité) et nourris avec une alimentation standard.

(1) Reprint requests to: Dr. E. Vanhems, Labo. d'Histologie U.E.R. 3, Université de Bordeaux II, 146, rue Léo-Saignat, 33076 Bordeaux. 
Les femelles dysgénésiques sont sacrifiées à l'âge de 1 mois, 48 jours, 2 mois, 5, 6 et 7 mois ef 12 mois; le sang, les hypophyses, les ovaires et leurs effecteurs sont rapidement prélevés.

Les hypophyses sont fixées, pendant $5 \mathrm{~h}$, au mélange de Bouin Hollande additionné de 10 p. 100 d'une solution saturée de chlorure mercurique ( $\mathrm{HgCl}$, sublimé), lavées $24 \mathrm{~h}$, à l'eau courante, déshydratées et incluses à la paraffine. Les coupes transversales, sériées, de $5 \mu$ d'épaisseur, sont montées à l'eau gélatinée. Après déparaffinage et réhydratation elles sont étudiées en immunofluorescence selon la technique indirecte. Nous avons utilisé l'anticorps anti-LH $\beta$ porcine, car il révèle la même catégorie cellulaire que les anticorps anti-LH totale de Rat et anti-FSH ovine ef présente la réaction la plus intense. La spécificité de ces anticorps a été étudiée dans des travaux antérieurs (Dubois et Dubois, 1974). La fixation de l'anticorps est révélée par des $\gamma$-globulines de Mouton anti- $\gamma$ globulines de Lapin conjuguées à l'isothiocyanate de fluorescéine. Les préparations sont contre-colorées au bleu Evans à 1/10 000 pour améliorer le contraste, enfin examinées sur un microscope Leitz équipé pour la fluorescence.

Une étude parallèle est effectuée sur des hypophyses de Rattes normales, servant de témoins, âgées de 1 mois et pubères âgées de 3 mois. Dans ce dernier cas, nous avons examiné les quatre stades du cycle œstral. Enfin, un dernier groupe est constitué de Rattes normales pubères castrées. La castration est effectuée trois semaines avant le prélèvement afin d'obtenir une stabilisation de la sécrétion gonadotrope au niveau le plus élevé.

Les cellules immunoréactives ont été dénombrées et leur densité estimée par rapport à un champ arbitraire, identique pour toutes les coupes. La proportion des cellules dites «de castration » (correspondant à la vacuolisation du cytoplasme des cellules gonadotropes) par rapport à l'ensemble de la population gonadotrope a été effectuée dans les mêmes conditions.

TABLEAU I

Efude, par immunocytologie, des cellules gonadotropes

dans les adénohypophyses de femelles normales et dysgénésiques

Estimation relative de l'abondance ef de la dimension des cellules

\begin{tabular}{|c|c|c|c|c|c|}
\hline & Age des femelles & $\begin{array}{c}\text { Nombre d'hypophyses } \\
\text { examinées }\end{array}$ & $\begin{array}{l}\text { Abondance } \\
\text { des cellules }\end{array}$ & $\begin{array}{c}\text { Cellules } \\
\text { de castration }\end{array}$ & $\begin{array}{l}\text { Dimension } \\
\text { des cellules }\end{array}$ \\
\hline Témoins & $\begin{array}{c}1 \text { mois } \\
3 \text { mois (Pro-oestrus) } \\
3 \text { mois } \\
\text { (castrées à l'âge } \\
\text { de } 70 \text { jours) }\end{array}$ & $\begin{array}{l}10 \\
10 \\
10\end{array}$ & $\begin{array}{l}+ \\
+ \\
+\end{array}$ & $\begin{array}{l}- \\
- \\
+\end{array}$ & $\begin{array}{c}+ \\
+ \\
+++\end{array}$ \\
\hline $\begin{array}{l}\text { Dysgéné- } \\
\text { siques }\end{array}$ & $\begin{array}{r}1 \text { mois } \\
48 \text { jours } \\
2 \text { mois } \\
5 \text { mois } \\
6 \text { mois } \\
7 \text { mois } \\
12 \text { mois }\end{array}$ & $\begin{array}{l}10 \\
10 \\
10 \\
10 \\
10 \\
10 \\
10\end{array}$ & $\begin{array}{c}++ \\
+++\dot{\mathrm{a}}++++ \\
+++\dot{\mathrm{a}}++++ \\
++\dot{\mathrm{a}}+++ \\
++\dot{\mathrm{a}}+++ \\
++\dot{\mathrm{a}}+++ \\
+\end{array}$ & $\begin{array}{c}-\dot{\mathbf{a}}++ \\
-\dot{\mathbf{a}}+++ \\
++++ \\
++++ \\
++++ \\
-\dot{\mathbf{a}}+\end{array}$ & $\begin{array}{c}++ \\
+++ \\
+++ \\
++++ \\
++++ \\
++++ \\
+\end{array}$ \\
\hline
\end{tabular}


La taille des cellules a été mesurée au planimètre intégrateur (Kontron-DigiplanISI). Les mesures ont été effectuées sur 100 cellules par échantillon.

Le sang recueilli sur héparine en vue des dosages de LH plasmatique est centrifugé ; les dosages sont réalisés par radioimmunologie sur le plasma décanté en utilisant le traceur, le standard et l'anticorps fournis par le NIAMD (National Insitute of Arthristis and Metabolic Diseases, Bethesda USA).

\section{Résultats.}

1) Immunocyfochimie de l'adénohypophyse. - Le nombre de cas étudiés, l'estimation relative de la quantité ef de la dimension des cellules gonadotropes, appréciées subjectivement, sont résumées dans le tableau 1. Dans le tableau 2 sont présentés les résultats de l'analyse cyłologique quantitative.

\section{TABLEAU 2}

Etude, par immunocytologie, des cellules gonodotropes dans les adénohypophyses de femelles normales et dysgénésiques. Analyse cytologique quantitative

\begin{tabular}{|c|c|c|c|c|}
\hline & Age des femelles & $\begin{array}{c}\text { Nombre de cellules } \\
\text { gonadotropes par } \\
\text { unité de champ }\end{array}$ & $\begin{array}{l}\text { Nombre de cellules } \\
\text { de castration par } \\
\text { unité de champ }\end{array}$ & $\begin{array}{c}\text { Surface cellulaire } \\
\text { en } \mu_{2}\end{array}$ \\
\hline Témoins & $\begin{array}{c}1 \text { mois } \\
3 \text { mois (Pro-œstrus) } \\
3 \text { mois } \\
\text { (castrées à l'âge } \\
\text { de } 70 \text { jours) }\end{array}$ & $\begin{array}{l}100 \\
125 \\
135\end{array}$ & $\begin{array}{l}0 \\
0 \\
2\end{array}$ & $\begin{array}{l}28 \pm 8 \\
30 \pm 10 \\
53 \pm 22\end{array}$ \\
\hline $\begin{array}{l}\text { Dysgéné- } \\
\text { siques }\end{array}$ & $\begin{array}{r}1 \text { mois } \\
2 \text { mois } \\
6 \text { mois } \\
12 \text { mois }\end{array}$ & $\begin{array}{r}170 \\
300 \\
210 \\
92\end{array}$ & $\begin{array}{r}4 \\
15 \\
110 \\
2\end{array}$ & $\begin{array}{l}34 \pm 12 \\
66 \pm 24 \\
77 \pm 31 \\
33 \pm 14\end{array}$ \\
\hline
\end{tabular}

L'examen des coupes d'hypophyses de sujets atteints de dysgénésie ovarienne révèle une hyperplasie et une hypertrophie constantes des cellules gonadotropes de 48 jours jusqu'à 7 mois, comparativement aux hypophyses de femelles normales, qu'elles proviennent de Rattes normales entières ou castrées (fig. 1 et 2).

Dès l'âge de 1 mois, les cellules fluorescentes sont nombreuses et grandes (fig. 3 ). A 48 jours et à 2 mois (fig. 4), on remarque une hyperplasie et une hypertrophie prononcées des cellules gonadotropes, plus ou moins accentuées selon les sujets, mais toujours très marquées. Les classiques cellules de castration apparaissent, mais leur présence n'est pas constante. En revanche, à 5, 6 et 7 mois (fig. 5), elles sont très nombreuses et on observe fréquemment une dégénérescence hypertrophique vacuolaire. Toutefois, les cellules fluorescentes sont dans l'ensemble moins abondantes qu'à 2 mois. A 12 mois (fig. 6), nous constatons une diminution très importante, et constante, du nombre des cellules gonadotropes. Parallèlement, les cellules de castration sont rarement observées. 
2) Dosage radioimmunologique de $\mathrm{LH}$ plasmatique. - Les concentrations de $\mathrm{LH}$ dans le plasma de femelles normales et dysgénésiques sont résumées dans le tableau 3. Dès l'âge de 1 mois, on constate que les taux de LH sont cinq fois plus élevés chez la femelle dysgénésique que chez la femelle normale. A partir de 2 mois et jusqu'à 7 mois, les taux sont très augmentés et correspondent aux valeurs trouvées chez les femelles normales castrées. A 12 mois, on observe une diminution spectaculaire du taux de LH plasmatique dont le niveau sécrétoire est identique à celui de femelles témoins entières.

TABLEAU 3

Toux de LH plasmatique

\begin{tabular}{|c|c|c|c|c|c|c|c|}
\hline \multicolumn{4}{|c|}{ Témoins } & \multicolumn{4}{|c|}{ Dysgénésiques } \\
\hline $\begin{array}{l}\text { Age des } \\
\text { femelles }\end{array}$ & 1 mois & $\begin{array}{c}3 \text { mois } \\
\text { (pro- } \\
\text { cestrus) }\end{array}$ & $\begin{array}{c}3 \text { mois } \\
\text { (castrées } \\
\text { à l'âge } \\
\text { de } 70 \text { jours }\end{array}$ & 1 mois & 2 mois & 6 mois & 12 mois \\
\hline $\begin{array}{l}\text { Nombre } \\
\text { d'animaux }\end{array}$ & 10 & 10 & 10 & 10 & 20 & 30 & 10 \\
\hline $\begin{array}{l}\text { Concentra- } \\
\text { tion de LH } \\
\text { en } \mathrm{ng} / \mathrm{ml} \\
\text { de plasma }\end{array}$ & $4,07 \pm 1,2$ & $33,8 \pm 8,5$ & $205,5 \pm 41$ & $24,9 \pm 16,2$ & $193 \pm 51,6$ & $241 \pm 62,6$ & $16,7 \pm 9,3$ \\
\hline
\end{tabular}

3) Caractères des effecteurs des stéroïdes. - Les images observées au niveau des organes cibles des stéroïdes (vagin, utérus) sont variables, en particulier jusqu'à l'âge de 5 mois. Certaines ne reflètent aucune imprégnation hormonale, d'autres (correspondant aux dysgénésies partielles) indiquent une stimulation du tissu cible (images de kératinisation ou de mucification vaginales: muqueuse utérine développée). Chez les animaux plus âgés, la réponse des effecteurs est plus homogène (épithélium vaginal pavimenteux, stratifié, souvent kératinisé); de plus, au niveau de la muqueuse utérine, on observe fréquemment une hypertrophie glandulo-kystique avec métaplasie pavimenteuse des glandes. Toutefois, quel que soit l'état de l'effecteur (donc la quantité de stéroïdes circulants), on n'observe de différence significative ni au niveau des cellules gonadotropes de l'hypophyse ni au niveau du taux plasmatique de $\mathrm{LH}$.

\section{Discussion.}

L'ensemble de ces résultats confirme, chez la Ratte atteinte de dysgénésie ovarienne, l'existence d'une hyperactivité gonadotrope que la technique histophysiologique des greffes avait déjà permis de mettre en évidence chez des sujets jeunes. L'hyperactivité gonadotrope est observée, quel que soit le degré de la dysgénésie (partielle ou totale) vérifiée par l'étude parallèle des ovaires et de leurs effecteurs. 
Jusqu'à l'âge de 2 mois, les observations en immunocytochimie, concernant l'abondance ef l'état des cellules gonadotropes, sont en accord avec l'importance de la dysgénésie : les cellules de castration n'apparaissent que dans les cas de dysgénésie totale, ef l'ensemble des cellules gonadotropes esł plus élevé en l'absence d'individualisation folliculaire dans l'ovaire. En revanche, les faux plasmatique de $\mathrm{LH}$ ne rendent pas compte de ces différences : les valeurs hormonales les plus élevées ne correspondent pas aux dysgénésies les plus graves. A 5, 6 ef 7 mois, la structure histologique de l'ovaire ne permet pas de connaître son passé (Vahnems et Bousquet, 1975a). Quel que soit l'état des effecteurs, les cellules gonadotropes sont toujours hypertrophiées, les cellules de castration sont très abondantes et les taux plasmatiques de LH sonf très élevés.

On peut expliquer cette riposte hypophysaire par une absence, ou une insuffisance, du taux des stéroïdes circulants, qui ne permet pas la régulation du feed-back hypothalamo-hypophysaire. En ce qui concerne les images de stimulation observées au niveau des organes cibles des stéroïdes, on peut penser qu'un taux faible, mais constant de stéroïdes (les frottis vaginaux sont acycliques) est capable de stimuler la cellule cible, mais insuffisant pour établir un feed-back au niveau central. En effet, on saił que si une muqueuse utérine hypoplasique reflèłe une sécrétion œstrogénique faible, en revanche, un endomètre hyperplasique, s'il témoigne parfois d'une activité ovarienne importante, peut aussi traduire une sécrétion normale ou même très faible d'œstrogène, mais de longue durée.

Dans le ças de dysgénésie partielle, l'hypothèse de l'incompétence des stéroïdes, au niveau hypothalamo-hypophysaire, doit aussi être envisagée pour expliquer l'emballement de l'hypophyse.

A 12 mois, la diminution spectaculaire des cellules gonadotropes ef du faux de LH plasmatique ef la disparition dans les limites de notre expérimentation (10 animaux) des cellules de castration, peuvent suggérer une sécrétion accrue des stéroïdes, devenue suffisante pour freiner les hormones hypophysaires. Mais il se peut aussi, qu'indépendamment des taux circulants des stéroïdes ovariens, l'hypophyse de sujets âgés ne soit plus apte à maintenir un niveau sécrétoire élevé.

Ces observations sont en accord avec les travaux récents d'autres auteurs (Howland ef Preiss, 1975 ; Howland, 1976 ; Gosden ef Bancroff, 1976) qui, dans plusieurs conditions expérimentales, ont observé que l'activité gonadotrope de l'hypophyse diminue avec l'âge. En particulier, après castration, ils ne trouvent plus les taux élevés caractéristiques de FSH et LH, chez des animaux âgés de 14 mois.

En conclusion, les différentes techniques utilisées nous ont permis de confronter les données histologiques et immunocytochimiques à celles des dosages radioimmunologiques. Elles conduisent à une convergence des résultats indiquant chez la Ratte dysgénésique jeune une hypersécrétion de gonadotropine égale, mais non supérieure, à celle observée chez les femelles témoins castrées. 


\section{Références}

DUBOIS P. M., DUBOIS M. P., 1974. Mise en évidence par immunofluorescence de l'activité gonadotrope LH dans l'antéhypophyse fœtale humaine, 37-62. In FOREST M. G., BERTRAND J., Endocrinologie sexuelle de la période périnatale, INSERM (Paris), vol. 32.

GOSDEN R. G., BANCROFT L., 1976. Pituitary function in reproductively senescent female rats. Exp. Géront., 11, 157-160.

HOWLAND B. E., 1976. Reduced gonadotropin release in response to progesterone or gonadotropin releasing hormone $(\mathrm{GnRH})$ in old female rats. Life Sci., 19, 219-224.

HOWLAND B. E., PREISS C., 1975. Effects of aging on basal levels of serum gonadotrophins, ovarian compensatory hypertrophy, and hypersecretion of gonadotrophins after ovariectomy in female rats. Fertil. Steril., 26, 271-276.

VANHEMS E., BOUSQUET J., 1971. Influence du Misulban sur le développement de l'ovaire du rat Ann. Endocrinol., 32, 753-761.

VANHEMS E., BOUSQUET J., 1973. Etude expérimentale chez le rat d'ovaires rendus dysgénésiques durant leur développement embryonnaire. Ann. Biol. animal. Bioch. Biophys., 13, Hors série, 79-88.

VANHEMS E., BOUSQUET J., 1975a. Evolution après la puberté et au cours de la sénescence d'ovaires de rattes rendus dysgénésiques par action prénatale du Misulban. Arch. Anat. Hist. Embr. norm. exp., 58, 121-132.

VANHEMS E., BOUSQUET J., 1975b. Ełude, chez le rat, de la fonction pituitaire de femelles dysgénésiques. Exploration par la méthode des greffes. C. R. Soc. Biol., 169, 1197-1200. 